\title{
Development and Testing of a Dual-Wavelength Sensitive Photopolymer Layer for Applications in Stacking of HOE Lenses
}

\author{
Sanjay Keshri ${ }^{1}$, Brian Rogers ${ }^{1,2}$, Kevin Murphy ${ }^{1}$, Kevin Reynolds ${ }^{1,2}$, Izabela Naydenova ${ }^{1,2}$ \\ and Suzanne Martin ${ }^{1, *}$
}

Citation: Keshri, S.; Rogers, B.; Murphy, K.; Reynolds, K.; Naydenova, I.; Martin, S. Development and Testing of a Dual-Wavelength Sensitive Photopolymer Layer for Applications in Stacking of HOE Lenses. Appl. Sci. 2021, 11, 5564. https://doi.org/ 10.3390/app11125564

Academic Editors: Maria

Antonietta Ferrara and

Principia Dardano

Received: 7 May 2021

Accepted: 10 June 2021

Published: 16 June 2021

Publisher's Note: MDPI stays neutral with regard to jurisdictional claims in published maps and institutional affiliations.

Copyright: (c) 2021 by the authors. Licensee MDPI, Basel, Switzerland. This article is an open access article distributed under the terms and conditions of the Creative Commons Attribution (CC BY) license (https:/ / creativecommons.org/licenses/by/ $4.0 /)$.
1 Centre for Industrial and Engineering Optics, Dublin City Campus, FOCAS Research Institute, School of Physics and Clinical \& Optometric Sciences, College of Science and Health, Technological University Dublin, 13 Camden Row, D08 CKP1 Dublin 8, Ireland; sanjayceloscusat@gmail.com (S.K.); brian.rogers@tudublin.ie (B.R.); kevin.p.murphy@tudublin.ie (K.M.);C17510763@mytudublin.ie (K.R.); izabela.naydenova@tudublin.ie (I.N.)

2 School of Physics and Clinical \& Optometric Sciences, College of Science and Health, Dublin City Campus, Technological University Dublin, Central Quad, Grangegorman Lower, D07 ADY7 Dublin, Ireland

* Correspondence: suzanne.martin@tudublin.ie

\begin{abstract}
Diffractive optical elements (DOEs) have been in development for many years and are an exciting technology with the capability to re-direct light, using diffraction rather than refraction. Holographic Optical Elements (HOEs) are a subset of diffractive optical elements for which the photonic structure is created holographically, i.e., by recording a specific interference pattern in a suitable, photosensitive optical material. Volume HOEs are of particular interest for some applications because of their very high diffraction efficiency and single diffracted order; however, high dispersion and angular wavelength selectivity still present significant challenges. This paper explores a method for producing a compound DOE useful for situations where elements designed for two separate target wavelengths can be advantageously combined to achieve a highly efficient HOE with reduced dispersion. A photopolymer material consisting of two independently sensitized laminated layers is prepared and used in sequential holographic recording at two different wavelengths. The photonic structures recorded are investigated through examination of their diffraction peaks and comparison with the structure predicted by modeling. Finally, the device is illuminated with an expanded diverging beam at both target wavelengths and with white light, and a strong diffracted beam is observed.
\end{abstract}

Keywords: diffractive elements; holography; photopolymer; holographic optical elements; volume gratings; compound optical elements; stacked gratings

\section{Introduction}

Diffractive Optical Elements (DOEs) are photonics structures that focus and redirect light, using diffraction rather than refraction. 'Volume' or 'thick' Diffractive Optical Elements utilize Bragg diffraction and can very efficiently transfer energy from the incident beam into a diffracted beam [1]. DOEs have the potential to replace optical components in a range of applications and have the advantages of being low cost, lightweight and in most cases, much thinner than their refractive counterparts.

The DOE element can be thought of as a set of localized gratings, each potentially having a different slant, orientation and spatial frequency (set by the patterning process) so that the direction and divergence of the diffracted output beam can be chosen as desired [2]. As a result, DOEs can replace standard optical components (e.g., lenses and mirrors) in some applications. Bragg diffraction, however, introduces wavelength and angular selectivity, which can be particularly restrictive if the element/grating is thick [1]. 
Photopolymer materials are typically prepared as thin, photosensitive films that polymerize when they absorb light, and can record patterns with very high spatial resolution. They can, therefore, be used to form volume DOEs in a low-intensity, single-exposure step, which is readily customized. Since the patterning step involves holographic recording, they are also often referred to as Holographic Optical Elements or HOEs. Currently, Diffractive Optical Elements are not routinely used with broadband sources. Some practical applications utilizing surface gratings in the management of light are reported in the literature; for example, LED light is re-directed through a $30^{\circ}$ angle by a binary diffractive lens [3] and broadband multicolor imaging is demonstrated by using multi-level surface holograms [4].

Fabrication of surface gratings is challenging, however, since it requires multiple manufacturing steps-lithography and etching - and the surface of the photonic structures is difficult to protect. Volume photopolymer HOEs [5] offer a significant improvement since they can be patterned (with low intensity exposure) [6] through the volume of the materials, can be laminated $[7,8]$ into a stack, and importantly, they can achieve near $100 \%$ re-direction of the incident light into a single output beam $[9,10]$. This optical patterning can potentially replace the etching and stamping process and facilitate customization and small batches. The main features of surface and volume HOEs are compared, at a glance, in Table 1.

Table 1. Comparing surface and volume diffractive optics elements.

\begin{tabular}{ccc}
\hline & Surface DOEs & Volume DOEs \\
\hline Fabrication & Multiple steps, including wet & single step (self-processing \\
phocessing & photopolymers) \\
Protection and Stacking & More difficult since the & Easier, the pattern is in the \\
pattern is on the surface & volume \\
Diffraction Efficiency & theoretical limit 33\% & Up to $100 \%$ \\
\hline
\end{tabular}

Research into beam patterning and shaping with HOEs has continued to advance [11-13], but solutions for broad wavelength sources are needed to increase the application range. Many sources, such as white light LEDs, output a set of wavelength ranges rather than a continuous broad band of wavelengths (as for solar radiation, for example). This paper explores a method for producing a compound element useful for situations where elements that are designed for two separate target wavelengths can be advantageously combined to achieve a highly efficient HOE. The authors previously reported stacked gratings for operation in broader wavelength range by calculating the correct angle of diffraction for each target wavelength and recording at a single wavelength in green in two separate layers that are then stacked after recording [14]. This approach is somewhat limited by the complex modeling and recording arrangements needed for elements such as lenses, as they contain a wide range of spatial frequencies and slants that need to be matched to the different wavelength, as well as the difficulty of assembling the stack after recording without introducing damage and/or shifts in the slant angle.

This paper presents a method of stacking that utilizes selectively pre-sensitized layers stacked together so that dual wavelength recording can take place independently in the two stacked layers and explores the process as a means to combine lens elements. The recordings are independent of one another because the dye sensitizer in each layer is matched to one of the recording laser wavelengths. It is demonstrated here for two layers but has the potential to be developed for additional layers (e.g., red, green, blue).

The stacking/multiplexing approach to correcting for chromatic dispersion is conceptually simple and relies on fabrication and combination of devices tailored to specific wavelength ranges. It has been exploited to multiplex or de-multiplex in diffusers [15] and lenses [16], in stacking meta-surfaces [17], and more recently, to wavelength multiplexing in lens arrays for full color imaging [18] with each layer fabricated of different materials and with different design parameters to optimize it for a specific frequency band. However, these devices are fabricated through lithographic processes or holographically recorded 
into a single layer of a pan-chromatic material through multiplexing. Here, we utilize the limited wavelength sensitivity range in the pre-stacked photopolymer layers to exploit the simple full field exposure step to holographically record a compound element at two separate wavelengths, without the need for complex modeling and fabrication techniques.

In the literature, a variation of dye concentration in multilayer photopolymer was used to improve the uniformity in volume gratings [19]. Three dyes were combined in a single layer of photopolymer material for full color display holography [20]. In this work, two photopolymer layers sensitive to two different wavelengths were laminated together and a lens was recorded in each layer by their designed wavelength in sequential manner.

Firstly, a blue-sensitive photopolymer formulation, previously developed for the recording of reflection holograms (at high spatial frequency), was optimized for the low spatial frequency and high layer thickness needed in the proposed off-axis collimating lens transmission-format element. Next, the design considerations for stacked lenses were discussed for the application of off-axis, high-diffraction efficiency collimating HOE elements. Finally, the dual-wavelength sensitive multi-layer structure was prepared by stacking two photopolymer layers and used to successfully record collimating elements for blue and green wavelengths. The local diffraction characteristics of the recorded microstructure were tested and limitations discussed.

\subsection{Concept}

Figure 1 shows the basic concept for stacking by recording in a dual-wavelength sensitive multi-layer structure. Holographic recording of lenses is achieved through the interference of two beams that mimic the input and output beams of the lens device. The interference pattern created is recorded in the volume of the photosensitive material as a periodic structure in which the spatial frequency and slant at a particular point are defined by the two wave-vectors normal to the interfering wave fronts at that point. This structure diffracts incoming light of the same wavelength and incident angle to re-create the original beams. In this case, a dual layer is prepared in which each layer responds optimally to a limited range of wavelengths in blue or green. This means that two sequential recordings in blue and green create a stack of two lenses, without the need for repositioning or adjustment of the layer. Due to the high selectivity of volume diffractive lenses, each lens reconstructs only the appropriate wavelength range and the output beams are identical for both wavelengths. This approach has potential in reducing chromatic dispersion in HOE lenses.

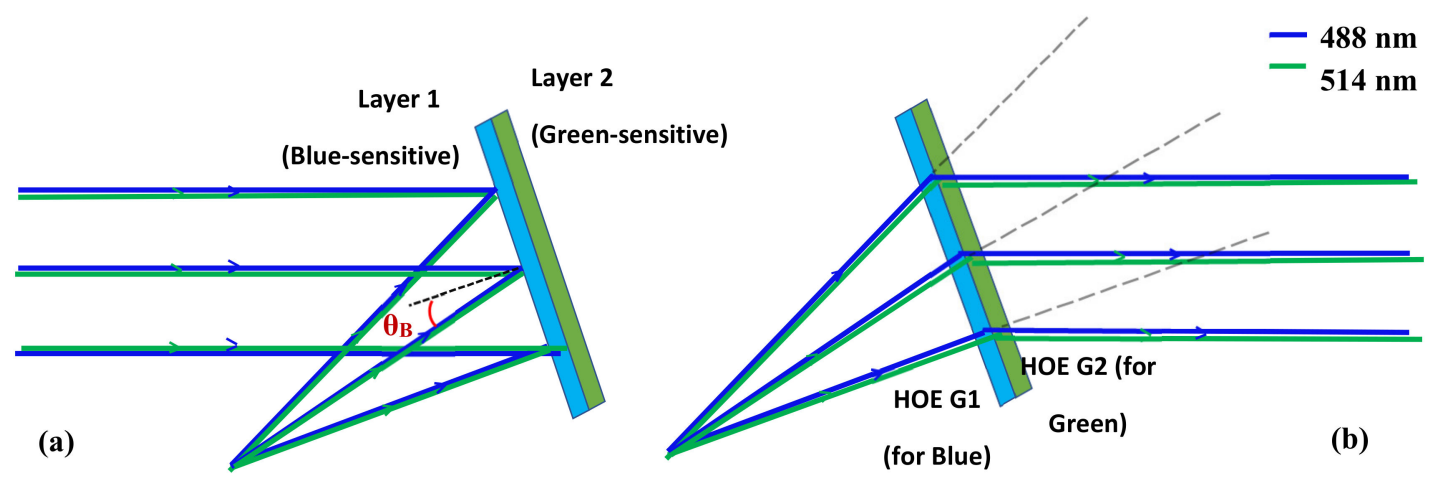

Figure 1. Schematic diagram for (a) recording two lens patterns, using two different wavelengths in a dual-sensitive layer to create stacked lenses. (b) Replay process showing that the stacked lenses reproduce the same collimated beam for both input wavelengths. 


\subsection{Theory}

The characteristics of a beam diffracted from a grating depend not only on its orientation and spatial frequency (photonic structure), but also on the angles and wavelength of the incident beam.

The classical grating equation for transmission [21] can be expressed by Equation (1):

$$
k_{i} \sin \theta_{0}+k_{d} \sin \theta_{+1}=\frac{2 \pi m}{n \Lambda}
$$

where, $k_{i}=\frac{2 \pi}{\lambda_{0}}$ and $k_{d}=\frac{2 \pi}{\lambda_{1}}$ are the propagation vector's modulus for the incident and diffracted beams, $\mathrm{m}=$ order of diffraction ( $m=1$ for volume HOE), $\theta_{0}=$ the angle of incidence in air, $\theta_{+1}=$ angle of diffraction in air, $n=$ refractive index of the medium, and $\Lambda=$ period of the refractive index modulation (spatial period).

Volume HOEs work on the principle of Bragg's law [22], and at the Bragg condition, the angle of incidence equals the angle of diffraction $\left(\theta_{+1}=-\theta_{0}\right.$ and $\left.\lambda_{0}=\lambda_{1}\right)$. Equation (2) defines the wavelength at the Bragg condition.

$$
\lambda=2 n \Lambda \sin \left(\frac{\theta_{+1}-\theta_{0}}{2}\right)
$$

In applications where the re-direction of illumination is important, volume diffractive optics are preferred because of their potential for high diffraction efficiency, meaning that a large proportion of the incident light is diffracted into a single diffraction order in a direction defined by 2 . The diffraction efficiency achieved also depends on the material characteristics of the grating, such as refractive index modulation and thickness. The Kogelnik Coupled Wave Theory (KCWT) [1] is a well-established tool for modeling the efficiency of such elements and can predict how the efficiency of Volume HOEs depends on many parameters, such as the illuminating beam incidence angle, replay wavelength, index modulation and grating thickness.

KCWT shows that at Bragg incidence, the diffraction efficiency for the volume grating can be modeled using the following:

$$
\eta=\operatorname{Sin}^{2}\left(\frac{\pi n_{1} d}{\lambda \operatorname{Cos} \theta_{B}}\right)
$$

where $\theta_{B}=$ Bragg angle inside the layer, $\mathrm{d}$ is the thickness of the grating and $\mathrm{n}_{1}$ the refractive index modulation.

The $Q$ factor is used to check whether the grating will function as a volume Bragg grating [23].

$$
Q=\frac{2 \pi \lambda d}{n \Lambda^{2}}
$$

With the parameters defined as above, a $\mathrm{Q}$ factor of $>10$ is considered to be in the 'volume' regime, and therefore is expected to have a single diffraction order and behavior that can be predicted using KCWT.

\section{Materials and Methods}

The dual-sensitive recording approach was explored here through a combination of modeling, experimental work, materials optimization, fabrication and testing. Firstly, the approximate diffractive lens device requirements (spectral range, thickness, spatial frequency range, refractive index modulation) for each layer were determined though modeling. Then, a previously developed blue-sensitive photopolymer formulation was optimized for the spatial frequency range and thickness needed here. This was achieved through a study of gratings holographically recorded in dry layers coated on glass slides and optically patterned using different exposure energies and inter-beam angles in the overlapping beams. Finally, holographic lens elements were recorded, using both blue $(488 \mathrm{~nm})$ and green $(514 \mathrm{~nm})$ wavelengths separately and together (in the dual-sensitive 
layer stack), and the resulting HOEs were studied through the analysis of theory diffraction behavior, allowing the recorded microstructure to be studied.

\subsection{Materials Preparation}

Two separate photopolymer formulations were prepared. First, a polyvinyl alcohol stock solution was prepared as described elsewhere [14] (10 g dissolved into $100 \mathrm{~mL}$ ). Two separate dye solutions were then prepared, both with $0.11 \mathrm{~g}$ of powdered dye (erythrosine $\mathrm{B}$ or acryflavin) in $100 \mathrm{~mL}$ of deionized water. Next, to make the green-sensitive formulation, the electron donor (triethanolamine $(2 \mathrm{~mL}))$ and the monomers $(0.6 \mathrm{~g}$ acrylamide and $0.2 \mathrm{~g}$ methylene bisacrylamide) were added to $17.5 \mathrm{~mL}$ of the PVA solution and stirred for one hour. Finally, $4 \mathrm{~mL}$ of the erythrosine B solution was added. For the blue-sensitive formulation, triethanolamine $(2 \mathrm{~mL})$ and the monomers $(0.8 \mathrm{~g}$ acrylamide and $0.2 \mathrm{~g}$ methylene bisacrylamide) were added to $17.5 \mathrm{~mL}$ of the PVA solution and stirred for one hour. Finally, $3 \mathrm{~mL}$ of the acryflavin solution was added.

Layers were prepared by using a micropipette to deposit a controlled amount of solution onto either glass microscope slides or high optical quality plastic of the same dimensions on a level surface. After allowing them to dry in a darkroom for 1-2 days, the dual-sensitive layer photopolymer was prepared by laminating the two layers together. For this step, it was important that one of the layers was prepared on a flexible substrate, in this case, Makrolfol of $0.375 \mathrm{~mm}$ thickness (flexible glass is also very suitable [14]). This allows for lamination of the two photopolymer surfaces together in a stable layer with no air gaps or bubbles.

\subsection{Optical Patterning: Holographic Recording Setup}

An off-axis holographic lens can be recorded by using the setup shown in Figure 2. Two collimated beams are arranged such that they overlap on the photopolymer layer, then a suitable lens is inserted into one of the beams such that it focuses the beam before it reaches the photopolymer as shown. This produces a diverging beam to interfere with the collimated reference beam and a HOE lens is recorded. The numerical aperture of the lens determines the divergence of the beam and the range of the angles of incidence at the photopolymer. This kind of recording was demonstrated previously with both cylindrical and spherical lenses [24]. However, in this instance, a cylindrical lens was used so that the beam only diverged in one axis (horizontal). This simplified the modeling and experimental testing since the grating characteristics varied in only one axis. In the blue sensitive photopolymer formulation optimization work, simple gratings were recorded, and the set-up was identical except for the fact that both beams were collimated, as the cylindrical lens was not present. For some characterization work, a $532 \mathrm{~nm}$ and a $473 \mathrm{~nm}$ laser were used but for recording in the dual-sensitized layer, an argon ion was used so that both the $514 \mathrm{~nm}$ and $488 \mathrm{~nm}$ laser lines could be used in the same optical setup.

When recording lenses, the range of angles of incidence which the diverging beam produces determines the inter-beam angles for the interference pattern, and consequently, the spatial frequency and slant at each location of the photopolymer. The cone of rays will be the same (assuming negligible aberrations) for recordings at both green and blue recording wavelengths. However, since the spatial frequency also depends on the wavelength of the incident light, this will lead to a different interference pattern for each recording. 


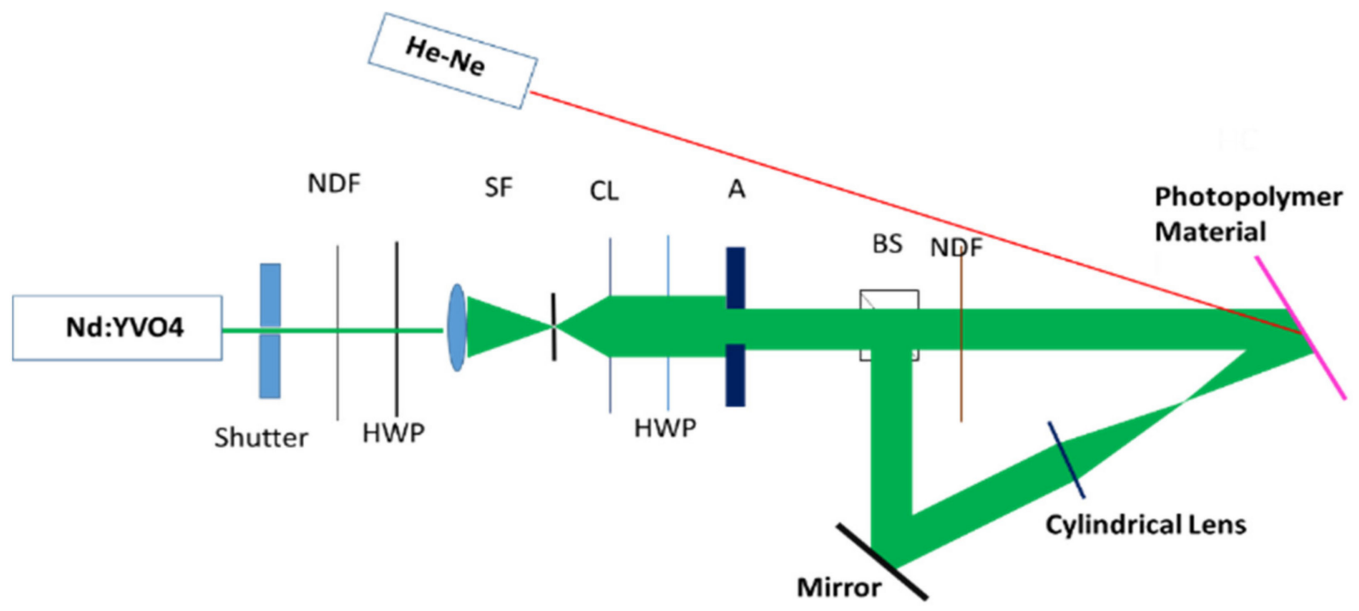

Figure 2. Experimental set-up for both the recording of a cylindrical holographic lens in green and probing using a He-Ne laser. NDF—neutral density filter; HWP—half-wave plate; SF-spatial filter; CL—collimator lens; A—variable aperture; BS-beam splitter.

\subsection{Testing the Recorded Lenses}

The recorded lenses were tested at three locations on the lens: at the center of the lens, $3 \mathrm{~mm}$ to the left of the center and $3 \mathrm{~mm}$ to the right of the center. Figure 3 illustrates how the Bragg positions for the two gratings vary because of the different wavelengths, and how they differ at the three probing positions. Testing involved illumination with an unexpanded He-Ne beam of about $1 \mathrm{~mm}$ in diameter and rotation of the sample through a wide angular range while monitoring the intensity changes in the transmitted or first-order beam. Since in all cases studied here, higher order beams are negligible, it was often more useful to monitor the zero order or transmitted beam so that the angular position, diffraction efficiency and FWHM information was available in a single scan. Except where otherwise stated, all tests were carried out in a computer-controlled system with a Newport rotation stage and photodetector and a $\lambda=633 \mathrm{~nm}$ He-Ne probe laser. Figure 3 shows an illustration of the different conditions for Bragg matching at three positions on the lens. The wave vectors for the input and output beams at both wavelengths are shown along with the grating vector for each position on the lens for each of the two wavelengths.

(a)
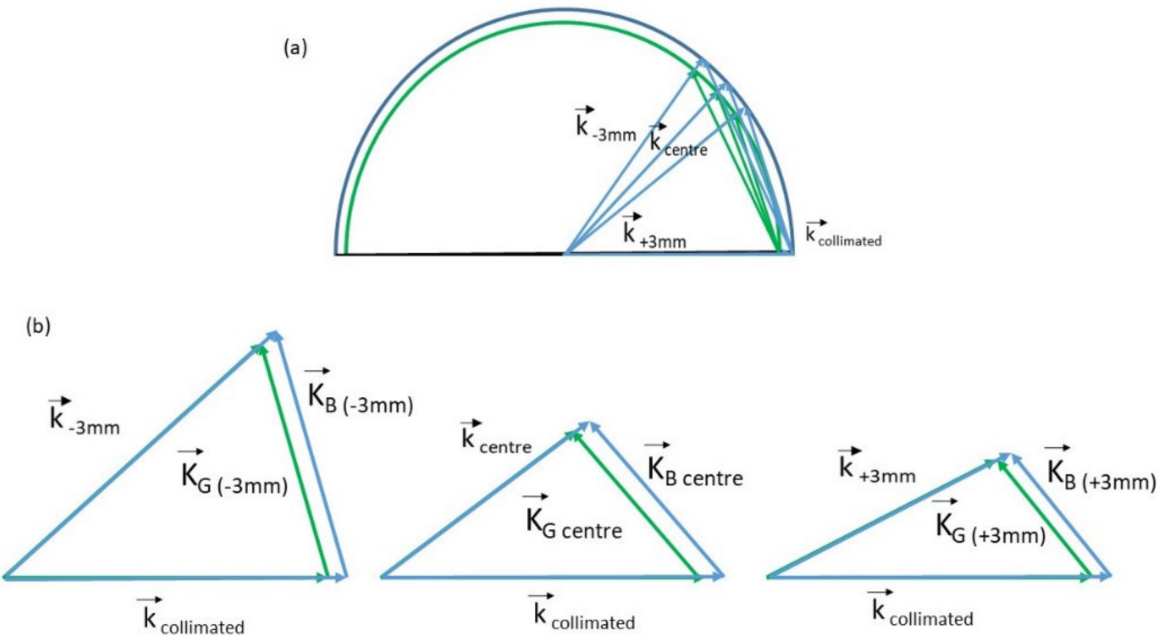

Figure 3. (a) Ewald Sphere illustration of the different conditions for Bragg matching at three positions on the lens and (b) Bragg matching condition for each position, $-3 \mathrm{~mm}$, center and $+3 \mathrm{~mm}$ shown separately for clarity. 


\subsection{Modeling the Recorded Microstructure-Calculation of Slant and Spatial Frequency}

In order to fabricate a HOE lens which collimates an off-axis diverging beam, a photonic structure is needed that will be on-Bragg everywhere for rays diverging from the off axis focal point and will re-direct them (through diffraction) into a collimated beam with a defined angle with respect to the HOE plane. At each location, the desired input beam angle can be defined, using the position of the focal point relative to that location. The desired output beam angle is known and is the same everywhere on the element (for the collimated output). Once the angles of the two beams, $\theta_{1}$ and $\theta_{2}$, are known, the slant angle $\left(\theta \mathrm{s}=\left(\theta_{2}+\theta_{1}\right) / 2\right)$ and Bragg angle $\left(\theta_{\mathrm{B}}=\left(\theta_{2}-\theta_{1}\right) / 2\right)$ at that location are determined from geometry, and the spatial frequency needed to obtain such a Bragg angle at the design wavelength is calculated from Equation (2) above. Figure 4 depicts this procedure for a single point on the DOE lens. Once the slant angle and spatial frequency are known, one can calculate, for every point on the lens, the interference pattern required to fabricate the desired structure at a given wavelength. If the recording wavelength is close to the design wavelength, however, this process becomes extremely convenient, as $\theta_{1}$ and $\theta_{2}$ become the recording angles. Then the process involves using the desired input beam as one of the recording beams, made to interfere in the plane of the polymer material with the other recording beam, which has the characteristics of the desired output beam. The dual-sensitive polymer layer enables this convenient process to be exploited at two separate wavelengths in one device.

Beam 1

(Input Beam)

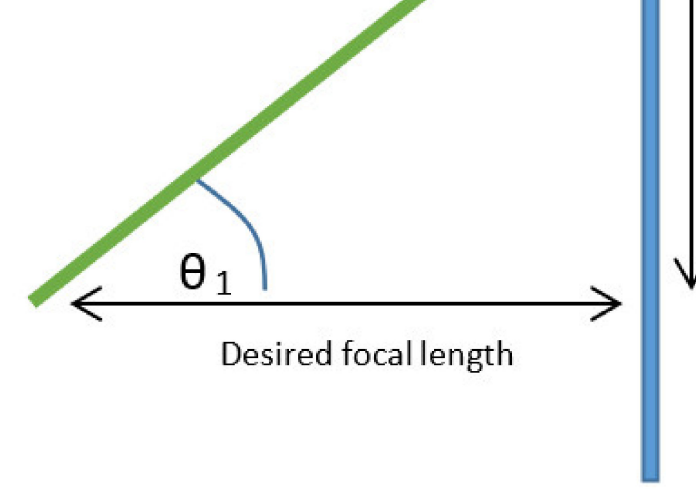

Distance from axis of focal point

Figure 4. Simple geometrical model used to calculate the required grating slant and spatial frequency for the grating needed to re-direct Beam 1 into Beam 2 (at each specific location on the DOE lens). For simplicity, refraction at the medium boundaries are not shown here, but are taken into account in the calculations. $\theta_{\mathrm{B}}$ is the Bragg angle; $\theta_{1}$ is the incident angle of the input Beam 1 and $\theta_{2}$ is the angle of the output Beam 2. $\theta_{\mathrm{S}}$ denotes the slant angle of the fringes. 


\section{Results}

\subsection{Modeling and Design of the Diffractive Optical Elements}

The stack envisaged consists of two off-axis lens elements, one designed for a blue target wavelength, and one designed for a green, both having a focal point of $5 \mathrm{~cm}$ from the plane of the photopolymer element.

The design considerations are as follows:

- Photonic structure: Each grating needs to have a tailored range of spatial frequency and slant to produce correct output angle (at that wavelength) for every point on the diffractive lens (Equations (1) and (2))

- Single diffracted beam: The design needs to ensure that each grating is functioning as a volume grating at all points (to avoid multiple diffracted beams) (Equation (4))

- Grating strength: Each grating must have a specific Refractive Index Modulation to produce high DE for its target wavelength (Equation (3))

- Avoiding crosstalk: Each grating must have a sufficiently narrow angular and spectral selectivity curve so that each grating will only re-direct light of the wavelength that it is designed for.

Target wavelengths [25] $514 \mathrm{~nm}$ and $488 \mathrm{~nm}$ were chosen to mimic typical green and blue peaks in a white LED spectrum for which close laser wavelengths were available for holographic recording. The design is based on high efficiency with the assumptions that higher diffracted orders are negligible, the grating is uniform though the depth of the polymer layer, and the polymer faithfully records the interference pattern (zero dimensional changes during recording).

As discussed above, each element consists of a range of grating spatial frequencies, which will change with the design wavelength, the off-axis angle and the aperture and focal length of the lens. In turn, the set of spatial frequencies recorded in the lens influences all of the above design considerations. On-axis elements are not suitable here because of well-understood issues around the limits of volume diffraction [2] that arise where the diffraction angle is very small (near zero spatial frequency). Off-axis elements are, therefore, used to focus light for these applications, so the first design step was to choose an offset angle, or central spatial frequency, that would avoid this. For transmission elements, high spatial frequencies (large offset angles) are less challenging to record so the limits of the low spatial frequency range are explored here.

\subsubsection{Green Lens Design}

First, the range of spatial frequencies expected to be recorded across the lens for a recording wavelength of $514 \mathrm{~nm}$ was calculated for a number of different off-axis angles (different central spatial frequencies). The calculated range of spatial frequencies for lenses having central spatial frequencies of 300, 400, 455 and $500 \mathrm{~L} / \mathrm{mm}$ are shown in Figure 5a. Figure $5 b$ shows the calculated $Q$ values for the lowest spatial frequency occurring in each lens from Figure $5 \mathrm{a}$ for different thicknesses. It was decided to choose the lens with the lowest off-axis angle (and spatial frequency range) that would ensure volume Bragg grating behavior $(Q>10)$ across the whole lens aperture for 150 microns in thickness. This ensures that higher orders are negligible. A minimum of 140-150 micron-thick gratings were shown to be needed to avoid crosstalk in previous work [14]; thickness above 150 microns can be more challenging to prepare without compromising layer optical quality. The lens chosen was, therefore, Lens 3 , with $150 \mu \mathrm{m}$ layer thickness and $455 \mathrm{~L} / \mathrm{mm}$ central spatial frequency. 


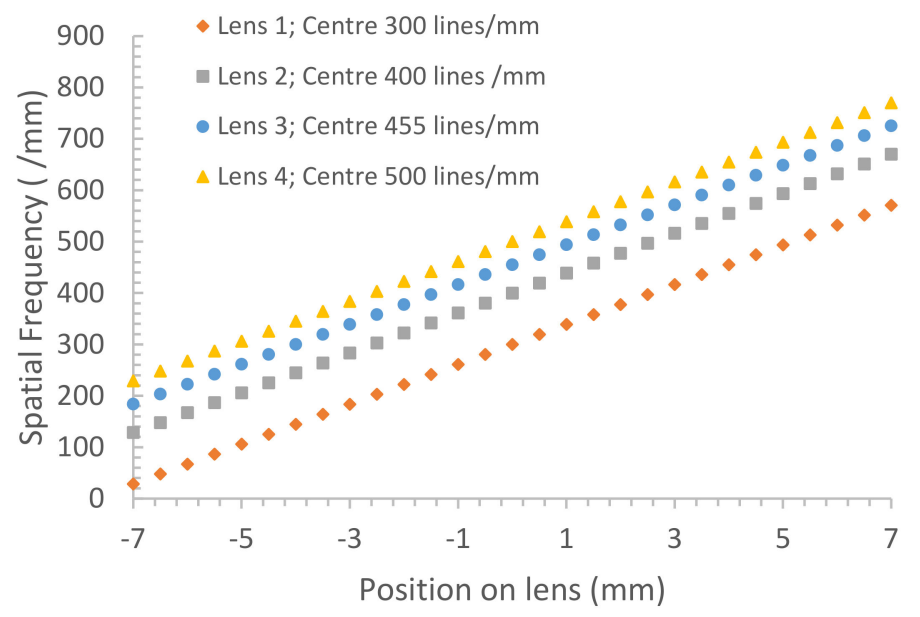

(a)

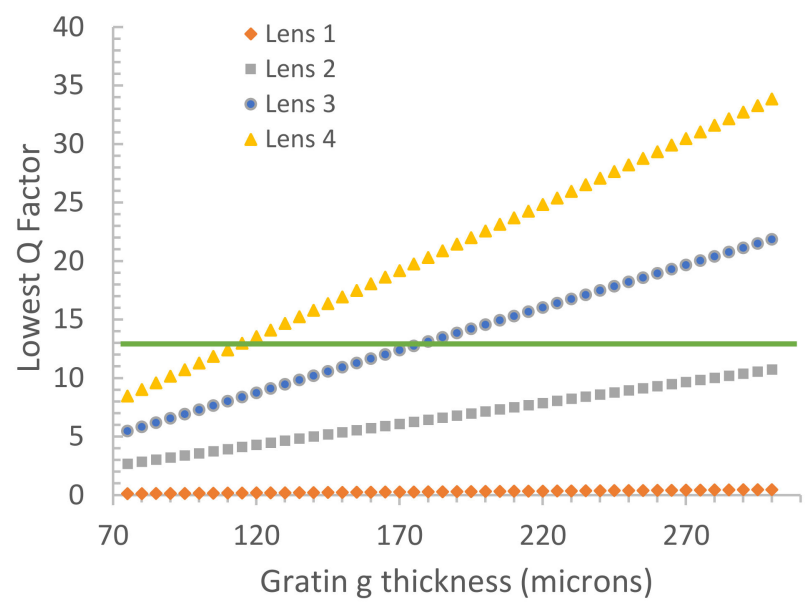

(b)

Figure 5. (a) Local grating spatial frequency across the diameter of four lenses with different central spatial frequencies, design wavelength $514 \mathrm{~nm}$, focal length $5 \mathrm{~cm}$, lens diameter $14 \mathrm{~mm}$ and (b) the $Q$ value for the lowest spatial frequency in each lens for a range of grating thicknesses. The green horizontal line indicates where $Q=10$, i.e., the minimum $Q$ value to ensure a single diffracted order.

\subsubsection{Blue Lens Design}

The next step was to design a second lens, which would produce the same output angle across the whole lens aperture for a $488 \mathrm{~nm}$ design wavelength with the same range of input and output angles. The $Q$ value for the lowest spatial frequency was 11.4, for a 150 micron layer, ensuring volume diffraction here, too. Since 150 micron layers were used, the capability of achieving sufficient grating strength was assured for the green sensitive formulation (100\% can, in fact, be achieved in much thinner layers [26]). Figure 6 shows the range of spatial frequencies across this lens, which has the same angular offset and focal length as Lens 3 but is designed for operation at $\lambda=488 \mathrm{~nm}$.

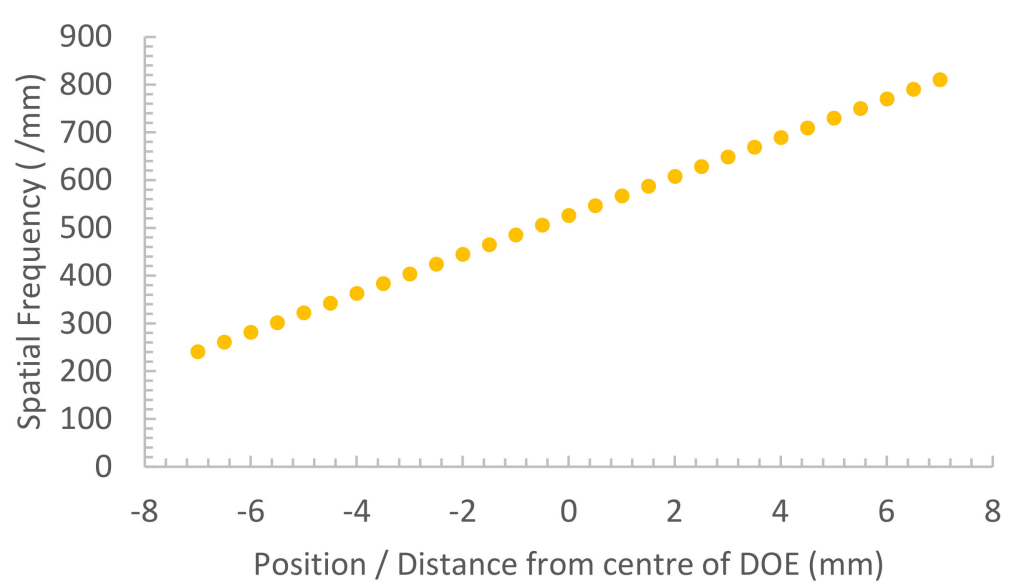

Figure 6. The range of spatial frequencies across the lens designed for $488 \mathrm{~nm}$, diameter $=14 \mathrm{~mm}$ with the same angular offset and focal length as Lens 3 but for operation at $\lambda=488 \mathrm{~nm}$.

The refractive index modulation needed to maximize the diffraction efficiency was controlled through exposure during recording, as detailed in the experimental section. Some characterization was needed in order to achieve this for the blue-sensitive formulation, which had not been as widely studied as the standard green (eythrosine B) formulation. 


\subsection{Optimization of the Acriflavine Based Photopolymer Layer}

In the literature, acriflavine sensitized photopolymer material was used in the development of a panchromatic photopolymer for holographic recording applications [20]. Previously, the characterization of a $60 \mu \mathrm{m}$ thick transmission grating with high spatial frequencies was reported [27]. In this work, a similar composition is optimized for the range of spatial frequencies polymer layer thickness needed in this application. Figure 7a shows the composition of the layers and Figure $7 \mathrm{~b}$, the absorption spectrum for this acriflavine-sensitized photopolymer.

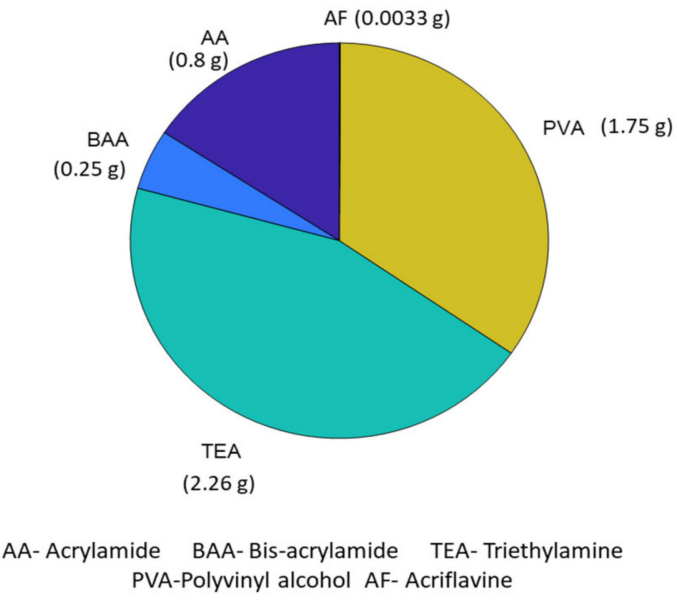

(a)

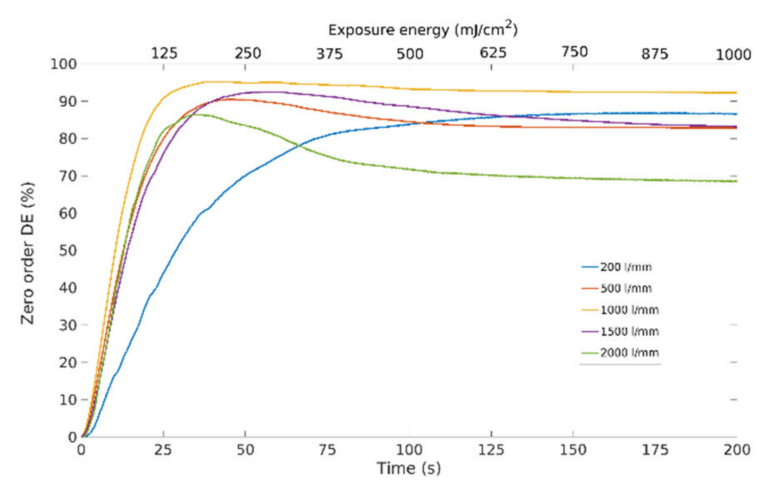

(c)

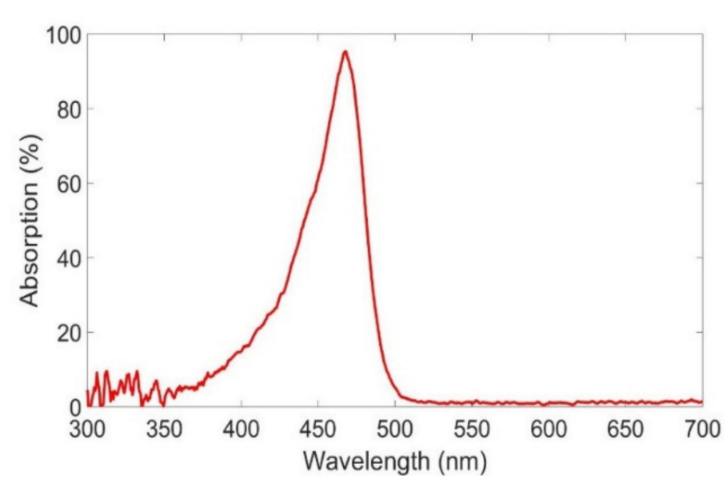

(b)

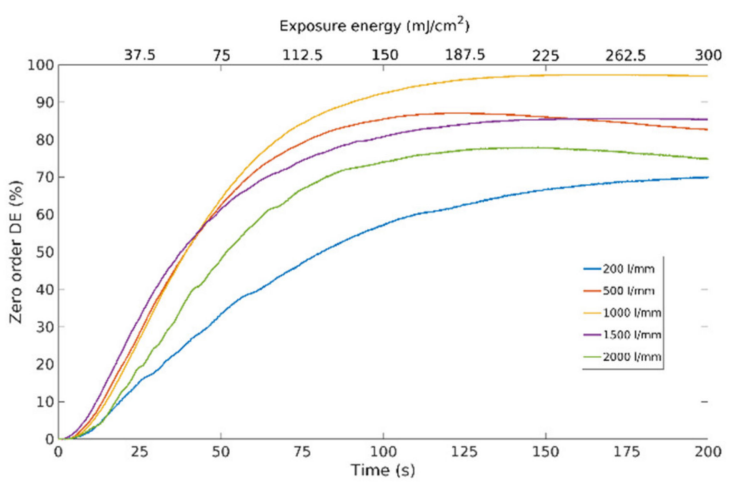

(d)

Figure 7. (a) The relative mass of the components of the acriflavine-sensitized dry photopolymer layer. (b) The absorption spectrum of the acriflavine-sensitized photopolymer (composition as outlined in (a)). (c) On-Bragg diffraction efficiency growth curve of $60 \mu \mathrm{m}$ thick gratings recorded at various spatial frequencies, with recording intensity $=5 \mathrm{~mW} / \mathrm{cm}^{2}(\mathrm{~d})$ with recording intensity $=1.5 \mathrm{~mW} / \mathrm{cm}^{2}$.

\subsubsection{Optimization for Spatial Frequency}

Figure 7 shows the optimization of the recording conditions for a range of spatial frequencies in Figure 7c,d. This study was carried out using 60 micron layers so that detailed information about the grating growth dynamics could readily be obtained using on-Bragg dynamic measurements of the diffraction efficiency during recording. High angular selectivity makes on-Bragg illumination during recording more challenging for 150 micron layers.

Using two different recording intensities, and monitoring on Bragg at $633 \mathrm{~nm}$, the recording dynamics were observed for spatial frequencies from 200 to 2000 lines/mm (equivalent to 5 micron to 0.5 micron fringe spacing). It should be noted that the zero-order 
transmission was monitored and the raw data from the zero-order transmittance were transformed into the first-order efficiency. In the diffraction efficiency curves the growth dynamics are very similar for all spatial frequencies across the range, except for the two extremes of 200 and 2000 lines/mm. At the higher end, the 2000 lines $/ \mathrm{mm}$ curves are observed to fall slightly below the mid-range curves. At 200 lines $/ \mathrm{mm}$, a significantly slower grating growth is observed, although the diffraction efficiency is similar after $200 \mathrm{~s}$ for the higher intensity recording. The photopolymer material composition and the absorption spectrum are also shown.

\subsubsection{Optimization for 150 Micron Thick Layers}

Real-time monitoring of the diffraction efficiency during grating recording is considerably more challenging at 150 microns, so a stepwise recording with multiple samples is sometimes needed to properly examine the grating growth, especially at higher spatial frequencies (due to the high angular selectivity). In this section, a combination of real time recording and multiple Bragg curves is used to study blue-sensitive layers of 150 micron thickness. Firstly, real-time recording was done for $200 \mathrm{~L} / \mathrm{mm}$ spatial frequency with higher $\left(5 \mathrm{~mW} / \mathrm{cm}^{2}\right)$ and lower $\left(1.5 \mathrm{~mW} / \mathrm{cm}^{2}\right)$ intensities. The growth curves of first-order efficiencies are shown in Figure 8a.

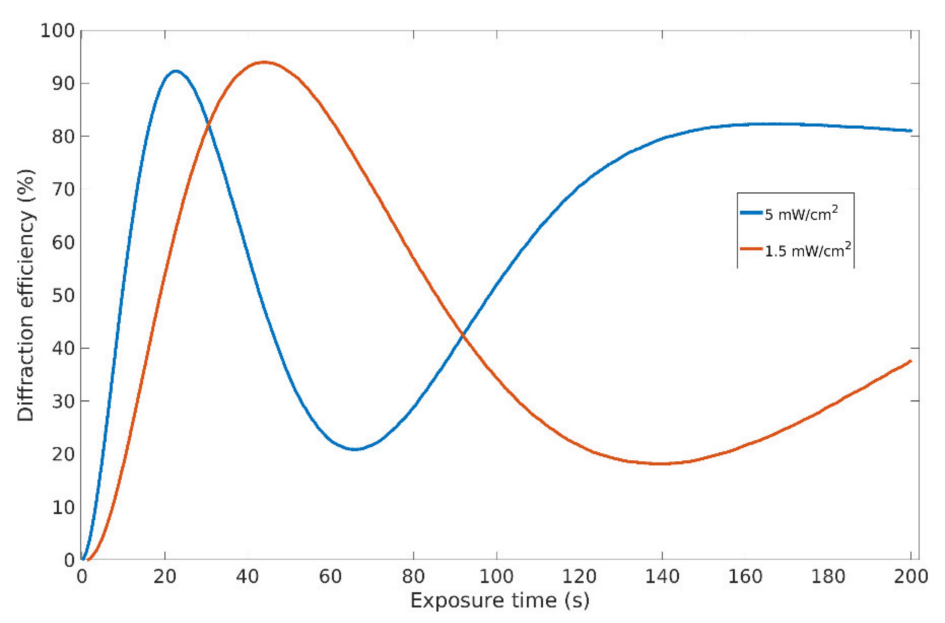

(a)

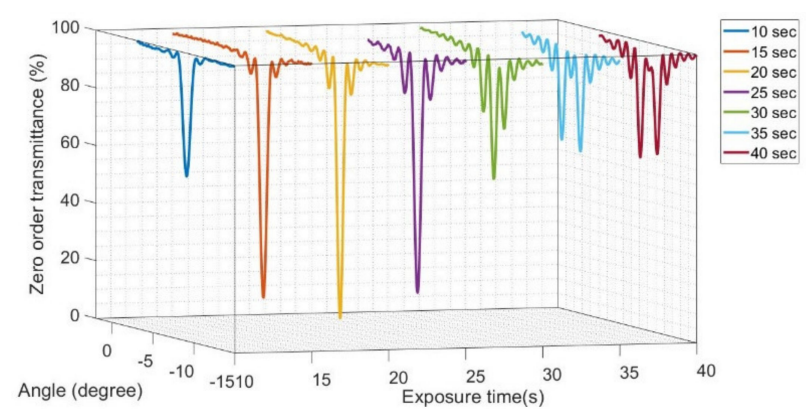

(b)

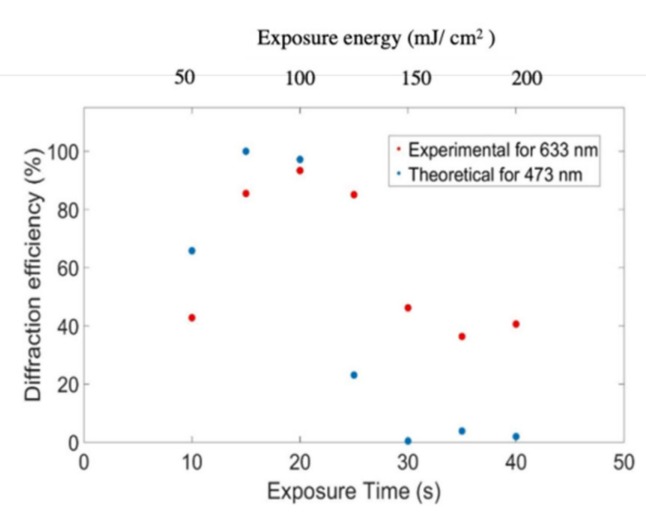

(c)

Figure 8. Diffraction efficiency growth for 150 micron thick blue-sensitive layers. (a) On-Bragg efficiency growth curve of for gratings recorded with spatial frequency $200 \mathrm{~L} / \mathrm{mm}$, (b) a series of zero-order Bragg selectivity curves for grating recorded with $500 \mathrm{~L} / \mathrm{mm}$, for a range of exposure times, measured at $633 \mathrm{~nm}$. (c) On-Bragg diffraction efficiency values obtained from $(\mathbf{b})$. The equivalent diffraction efficiency that would be expected at $488 \mathrm{~nm}$ is also shown so that the optimal recording time can be identified. 
For $500 \mathrm{~L} / \mathrm{mm}$, individual gratings were recorded at a constant intensity $\left(5 \mathrm{~mW} / \mathrm{cm}^{2}\right)$ but with different exposure times. The recording time for each gratin $\mathrm{g}$ was increased from 10 to $40 \mathrm{~s}$ with an interval of $5 \mathrm{~s}$. Figure $8 \mathrm{~b}$ shows the results obtained in the form of zeroorder Bragg selectivity curves, when the individual gratings were probed after recording by a He-Ne laser. Each curve graphs a set of data obtained by rotating the probe beam though a range of angles while monitoring the diffraction efficiency. The curves are arranged in Figure $8 \mathrm{~b}$ according to the exposure time used to create the grating. They show gratings of increasing strength up to the optimum exposure and then the characteristic indications of overmodulation (growing sidelobes). The minimum value from each zero-order Bragg selectivity was taken to obtain the growth curve, which is shown in Figure 8c, along with theoretical values for the efficiency, which is for the same strength grating at $488 \mathrm{~nm}$ (using Equation (3)). Considering the growth of diffraction efficiency for both $200 \mathrm{~L} / \mathrm{mm}$ and $500 \mathrm{~L} / \mathrm{mm}$ spatial frequencies, the optimized time to record gratings with $5 \mathrm{~mW} / \mathrm{cm}^{2}$ for $\lambda=488 \mathrm{~nm}$ is estimated $\approx 16-20 \mathrm{~s}$. As seen in Figure 6, the holographic lens to be recorded covers the range of spatial frequencies from $198 \mathrm{~L} / \mathrm{mm}$ to $790 \mathrm{~L} / \mathrm{mm}$.

\subsection{Optimization of the Holographic Recording Conditions for the Fabrication of Individual Lenses at Blue and Green Wavelengths}

The next step was to record and test the individual lenses. As lenses consist of many spatial frequencies and slant angles in a single element, it is necessary to determine the optimal recording conditions for each lens, as well as to analyze the overall diffraction behavior to ensure that the expected microstructure is recorded. This makes it possible to (i) confirm experimentally which exposure conditions (from the range identified above) give the best performance across the lens aperture and (ii) to provide performance data of individual lenses as a comparator for the stacked lenses.

\subsubsection{Recording and Characterization of Holographic Lens Designed for $488 \mathrm{~nm}$}

Individual lenses were recorded at a constant recording intensity with a number of different exposure times. Each lens was then characterized by probing it at three positions across the element through a wide angular range in order to measure the local $\mathrm{DE}$ and angular positions of the diffraction peaks (troughs). This allowed for fine tuning of efficiency and uniformity across the lens aperture as well as investigation of the local spatial frequency and slant of the recorded photonic structure. The spatial frequency at each location is indicated by the angle between the two diffraction maxima (minima) in the intensity values of the zero-order wide angular scan, and the slant can be estimated from their offset from $0^{\circ}$ at each location. For convenience, this is done at a single wavelength $(633 \mathrm{~nm})$.

The recording of these individual lenses was done at a constant recording intensity of $3.6 \mathrm{~mW} / \mathrm{cm}^{2}$ with 17,27 and $37 \mathrm{~s}$ exposure times, thereby achieving total exposure energies of 61,97 and $130 \mathrm{~mJ} / \mathrm{cm}^{2}$, respectively. After recording, the lenses were probed at three positions across the element (the center of the lens and $+/-3 \mathrm{~mm}$ either side). The zero order and first order intensity curves are shown in each case for a range of incident angles in Figure 9. The latter is useful because it is a direct confirmation that the light diffracted from the zero-order curve appears in the first order, validating the assumption that volume gratings/Bragg diffractions as beams other than those of the first-order are of negligible intensity. A number of observations can be made from these results. Firstly, the uniformity of the diffraction efficiency is an important factor in the performance of the collimating device so by looking at the diffraction behavior at three known locations, the recording can be optimized for uniformity as well as the magnitude of the diffraction efficiency. Secondly, the spatial frequency of the grating can be examined by studying the separation of the diffraction peaks/troughs. From Table 2 below, we can see that the separations expected for a $633 \mathrm{~nm}$ probe beam for the lens element G1 (designed for $488 \mathrm{~nm}$ light) are $13.4^{\circ}$, $18.0^{\circ}$ and $22.5^{\circ}$ respectively. Taking Figure $9 \mathrm{a}$ as an example, we observe separations of $12.1^{\circ}, 16.8^{\circ}$ and $22.8^{\circ}$. These match the expected values to within $1.2^{\circ}$. The differences are probably due to errors in positioning of the probe beam and an averaging effect caused 
by the probe beam spanning a non-zero range of locations/grating periods. There is also some variation between the three samples.
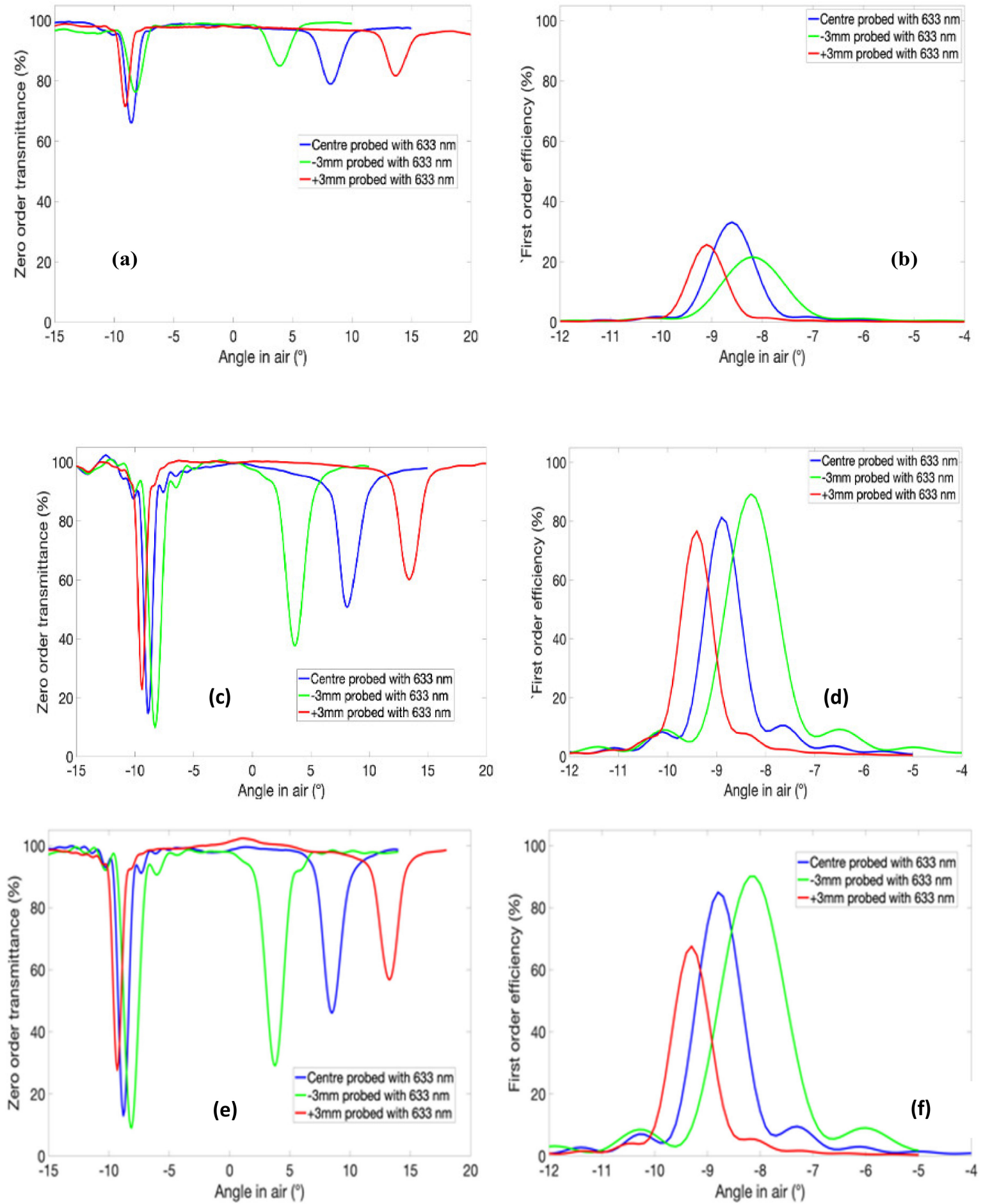

Figure 9. Experimental data for (a) zero-order transmission and (b) first-order diffraction efficiency at three points across the lens fabricated in acriflavine-sensitized photopolymer layer, using exposure $61 \mathrm{~mJ} / \mathrm{cm}^{2}$, exposure $97 \mathrm{~mJ} / \mathrm{cm}^{2}(\mathbf{c})$ and $(\mathbf{d})$ exposure $130 \mathrm{~mJ} / \mathrm{cm}^{2}(\mathbf{e})$ and (f). 
Table 2. The measured diffraction angle (angular separation of +1 and -1 diffraction orders) in comparison to the theoretical diffraction angles for the 4 samples at $633 \mathrm{~nm}$ wavelength.

\begin{tabular}{|c|c|c|c|c|c|c|}
\hline \multirow[t]{2}{*}{$\begin{array}{l}\text { Position on } \\
\text { Lens } \\
(\mathrm{mm})\end{array}$} & \multirow{2}{*}{$\begin{array}{c}\begin{array}{c}\text { Measured } \\
\text { Diffraction } \\
\text { Angle } \\
\text { (Degrees) }\end{array} \\
\text { Sample } 1\end{array}$} & \multirow{2}{*}{$\begin{array}{c}\begin{array}{c}\text { Measured } \\
\text { Diffraction } \\
\text { Angle } \\
\text { (Degrees) }\end{array} \\
\text { Sample } 2\end{array}$} & \multirow{2}{*}{$\begin{array}{c}\begin{array}{c}\text { Measured } \\
\text { Diffraction } \\
\text { Angle } \\
\text { (Degrees) }\end{array} \\
\text { Sample } 3\end{array}$} & \multirow{2}{*}{$\begin{array}{c}\begin{array}{c}\text { Measured } \\
\text { Diffraction } \\
\text { Angle } \\
\text { (Degrees) }\end{array} \\
\text { Sample } 4\end{array}$} & \multicolumn{2}{|c|}{$\begin{array}{l}\text { Theoretical Diffraction Angle for } \\
633 \mathrm{~nm} \\
\text { (Degrees) }\end{array}$} \\
\hline & & & & & $\begin{array}{l}\text { G1 Lens for } \\
488 \mathrm{~nm}\end{array}$ & $\begin{array}{l}\text { G2 Lens for } \\
514 \mathrm{~nm}\end{array}$ \\
\hline$-3 \mathrm{~mm}$ & 13.1 & 13.1 & 11.2 & 12.4 & 13.4 & 11.9 \\
\hline Center & 17.8 & 18.3 & 16.9 & 17.8 & 18.0 & 16.0 \\
\hline$+3 \mathrm{~mm}$ & 23.0 & 23.4 & 22.1 & 22.8 & 22.6 & 20.0 \\
\hline
\end{tabular}

\subsubsection{Recording and Characterization of Holographic Lens Designed for $514 \mathrm{~nm}$}

The process described in Section 3.3.1 was repeated for a holographic lens, designed to operate at $514 \mathrm{~nm}$, and recorded in an erythrosine-B-sensitized photopolymer layer. This time, the recording wavelength was $514 \mathrm{~nm}$ and recording energies of $98 \mathrm{~mJ} / \mathrm{cm}^{2}$ and $70 \mathrm{~mJ} / \mathrm{cm}^{2}$ were used. From Table 2 below, we can see that the separations expected for a $633 \mathrm{~nm}$ probe beam for the lens element G2 (designed for $514 \mathrm{~nm}$ light) are 11.9, 16.0 and 20.0. Taking Figure 10a as an example, we observe separations of $10.5^{\circ}, 15.0^{\circ}$ and $20.3^{\circ}$. These match the expected values to within $1.4^{\circ}$. However, the match appears to be poorer in Figure 10c.
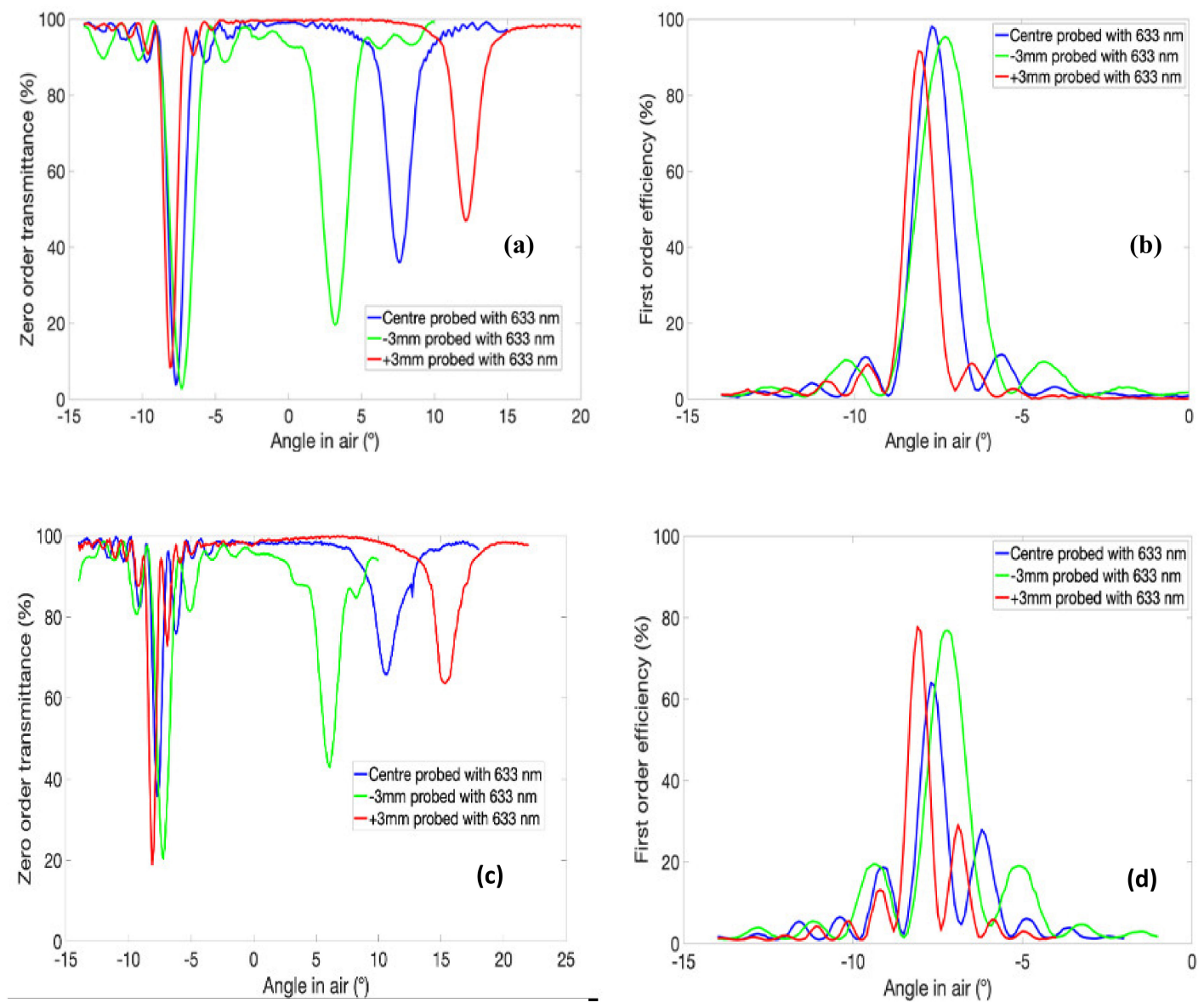

Figure 10. Experimental data for (a) the zero-order transmission and (b) the first-order diffraction efficiency at three points across the lens fabricated in a $150 \mu \mathrm{m}$ thick eythrosine-B sensitized photopolymer layer. (Exposure energy $=70 \mathrm{~mJ} / \mathrm{cm}^{2}$ ) and for (c) and (d) with exposure energy $=98 \mathrm{~mJ} / \mathrm{cm}^{2}$. 


\subsection{Two Lenses in Stacked in Dual-Sensitive Photopolymer Layer}

For the final device, the dual-sensitized layer was prepared as described above. Two lenses were then recorded sequentially using the same optical set-up (described above) but switching an Ar-Ion lasers lasing wavelength from $514 \mathrm{~nm}$ and $488 \mathrm{~nm}$ between the recording, using the lasers coupling prism. For each exposure, the intensity and duration followed the optimized conditions found in the previous sections. In the object beam, a conventional cylindrical lens was introduced to create a diverging beam in one axis, which interfered with a collimated reference beam. The resulting interference pattern was recorded in each photopolymer layer. Firstly, a laser $(\lambda=514 \mathrm{~nm})$ records the lens pattern in the erythrosine-B-sensitized photopolymer layer, which is the second layer in the stack. Secondly, the laser at $488 \mathrm{~nm}$ records the lens in the acriflavine-sensitized photopolymer material, which is the first layer in the stack. After recording, the stacked lenses were probed at three positions (center, $+3 \mathrm{~mm}$ and $-3 \mathrm{~mm}$ ), over a wide range of angles using a $633 \mathrm{~nm}$ wavelength laser to obtain the zero-order transmission angular selectivity curve. A set of four stacked lenses were recorded with the same optimized recording conditions (from Section 3.3) and their results are shown in Figure 11.

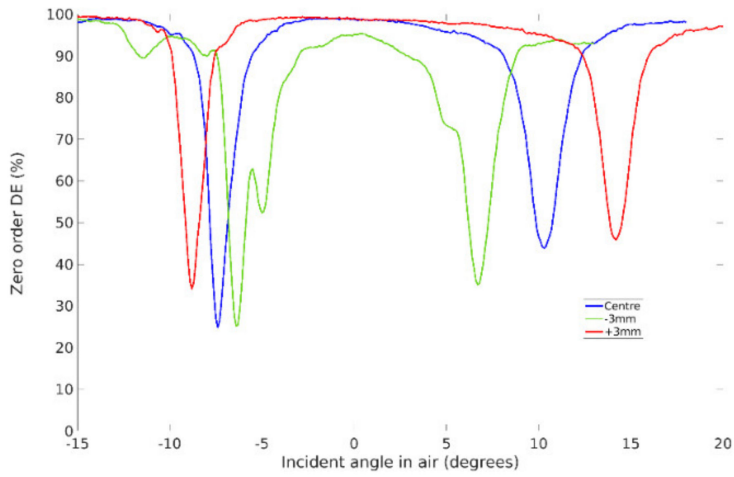

(a)

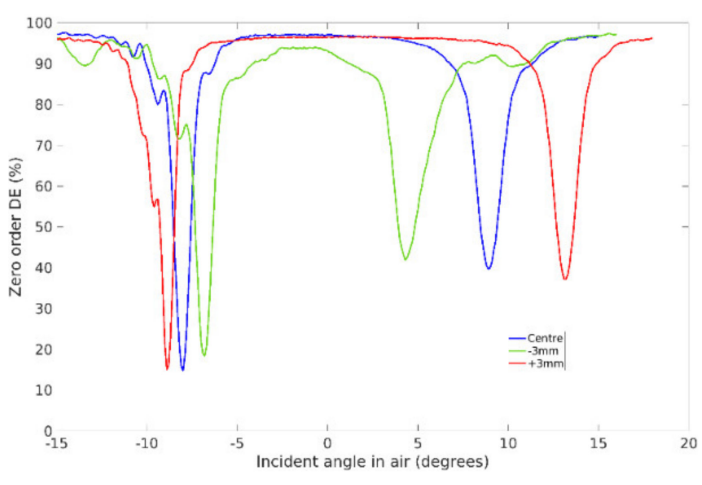

(c)

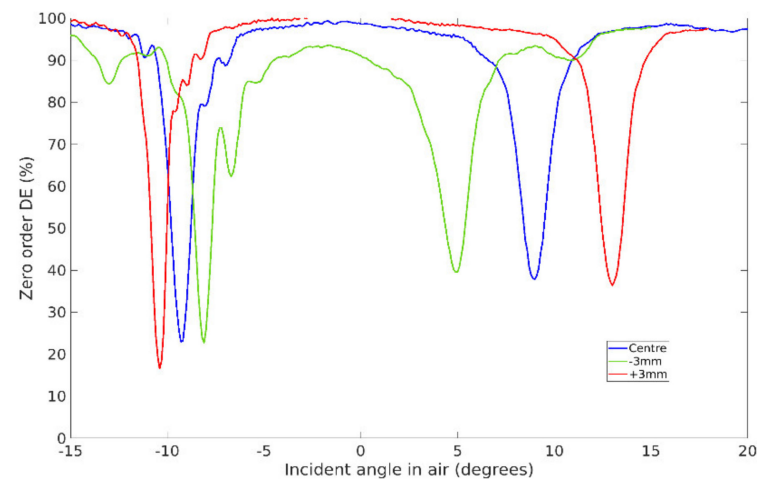

(b)

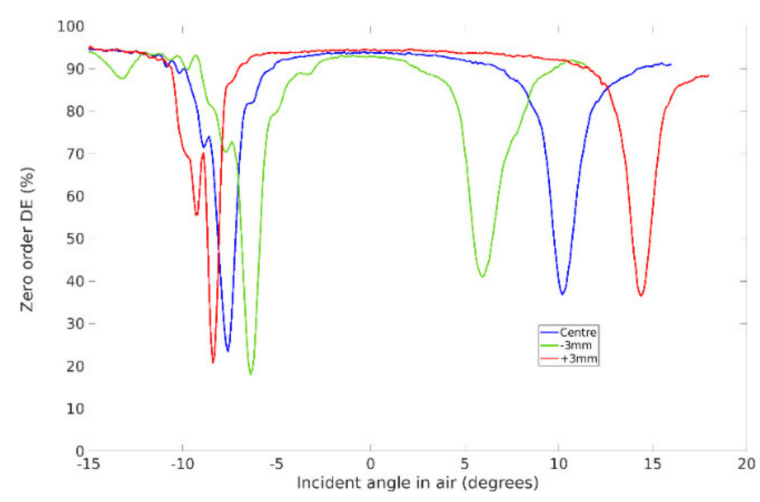

(d)

Figure 11. Zero-order transmission angular selectivity curves when the stacked lens is probed by a He-Ne laser at three positions. (a), (b), (c) and (d) show the results for samples (1), (2), (3) and (4) respectively.

In Table 2, the angular separation of the incident angles at which maximum diffraction occurs for $633 \mathrm{~nm}$ light is tabulated for the three positions in all four samples. A theoretical angular separation is also shown, based on the diffraction angle expected for the recorded spatial frequency at that location. Neither lens is designed for $633 \mathrm{~nm}$ light, which is why some secondary peaks were observed (not noted in the table). The peak positions are closer to the positions for the lens designed for blue light, except in the case of sample 2, possibly because this is the lens that the incident beam meets first in the stack. 
In order to examine the diffraction angles at the target wavelengths, sample 1 was then tested at the design/recording wavelengths. Using a manual rotation stage, the angular position of the diffraction peaks was obtained, and the angular separation or full diffraction angle was calculated. Table 3 shows the measured diffraction angle (angular separation of +1 and -1 diffraction orders) in comparison to the theoretical diffraction angles for both gratings in the stack while probing at the recording/design wavelengths. In most cases ( $+3 \mathrm{~mm} \mathrm{G1}$ is the exception) there is better than $0.5^{\circ}$ agreement between the measured diffraction angle for the stack with the expected diffraction angle for that wavelength.

Table 3. Table showing the measured diffraction angle (angular separation of +1 and -1 diffraction orders) in comparison to the theoretical diffraction angles for both gratings in the stack while probing at the recording/design wavelengths.

\begin{tabular}{|c|c|c|c|c|}
\hline \multirow[t]{2}{*}{$\begin{array}{l}\text { Probe Wavelength } \\
\text { (nm) }\end{array}$} & \multirow[t]{2}{*}{$\begin{array}{l}\text { Position on Lens } \\
\qquad(\mathrm{mm})\end{array}$} & \multirow[t]{2}{*}{$\begin{array}{c}\text { Measured Diffraction } \\
\text { Angle } \\
\text { (Degrees) }\end{array}$} & \multicolumn{2}{|c|}{$\begin{array}{c}\text { Theoretical Diffraction Angle } \\
\text { (Degrees) }\end{array}$} \\
\hline & & & $\begin{array}{l}\text { G1 lens designed for } \\
\text { blue } 488 \mathrm{~nm}\end{array}$ & $\begin{array}{l}\text { G2 lens designed for } \\
\text { green } 514 \mathrm{~nm}\end{array}$ \\
\hline \multirow[t]{3}{*}{514.5} & Center & 13.3 & 14.1 & 13.4 \\
\hline & $-3 \mathrm{~mm}$ & 10.0 & 10.5 & 10.0 \\
\hline & $+3 \mathrm{~mm}$ & 18.3 & 17.8 & 16.9 \\
\hline \multirow[t]{3}{*}{488} & Center & 13.0 & 13.4 & 12.8 \\
\hline & $-3 \mathrm{~mm}$ & 10.3 & 10.0 & 9.5 \\
\hline & $+3 \mathrm{~mm}$ & 17.0 & 16.9 & 16.0 \\
\hline
\end{tabular}

The stack was then illuminated with laser light. The input beam was a horizontally diverging beam produced by the cylindrical lens (the same lens that was used in recording). It was arranged that this be incident on the stack at the appropriate angle and the laser was then switched between wavelengths of $488 \mathrm{~nm}$ and $514 \mathrm{~nm}$. In both cases, an intense diffracted beam was clearly observed (to the right in Figure 12). While the transmitted portion of the input beam (seen on the left in each figure) was highly diverging, the diffracted output beam was observed to have much lower divergence and to have a circular cross-section. This demonstrates that the stack functions well for both wavelengths. As shown in Figure 12c, the process was repeated with a white light which was collimated and passed through the same cylindrical lens. Again, the stack produced a strong diffracted beam with a circular cross section (to the right in the image). Of course, some dispersion of the colors in the white light source is also evident, especially for the red and violet colors at the extreme ends of the visible spectrum.
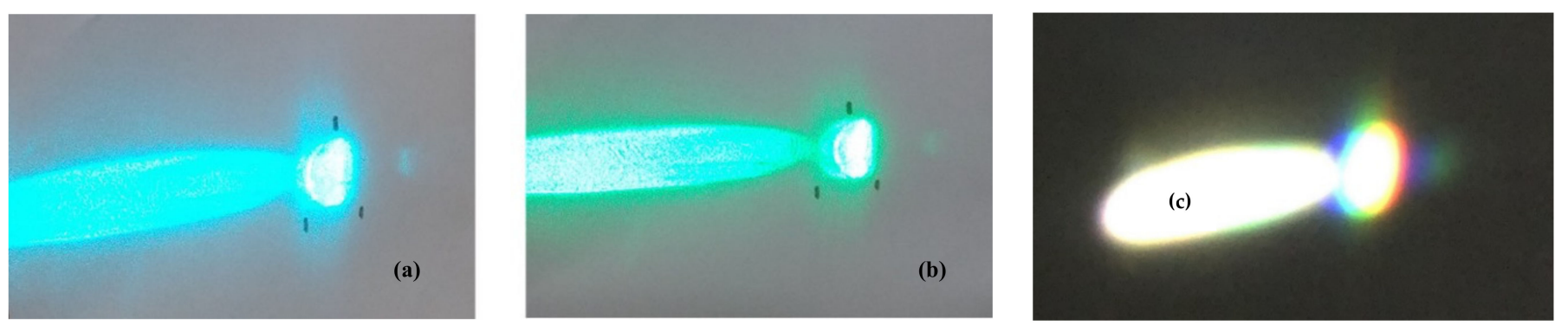

Figure 12. Demonstration of the stacked lens producing a collimated beam when illuminated with a cylindrically expanding laser beam at (a) $488 \mathrm{~nm}$ and (b) $514 \mathrm{~nm}$ and (c) with a white LED passing through a cylindrical lens.

\section{Discussion}

The exposure optimization work for blue recording showed the viability of using this blue-sensitized photopolymer for recording low spatial frequency gratings in layers as thick as 150 microns. High diffraction efficiencies were obtained with a range of exposures. This material had not previously been characterized in this range. The recording of the DOE 
lenses holographically in individual layers showed the challenges of equalizing efficiency across a DOE that contains a range of spatial frequencies, but $80-90 \%$ was achieved for green recording and greater than $90 \%$ for the blue recordings. With optimization, this could be improved further. It should be noted that the results indicate that two structures were recorded with the microstructure that matched well to the theoretical modeling. However, because the two gratings were designed to have overlapping input and output beams when they function properly, it is not possible to resolve the two individual sets of grating structures. Even at wavelengths far from the design wavelengths, modeling showed that the peaks for $\mathrm{G} 1$ and $\mathrm{G} 2$ would be less than $0.5^{\circ}$ apart. In addition, limitations in repeatable positioning of the probe beam on the lens meant that there were relatively large errors in the actual peak positions as well as some averaging over a range of neighboring locations. This will be improved by using a smaller diameter probe beam and devising a system of fiducial marks to properly locate the recording center.

\section{Conclusions}

In this paper, two photopolymer layers sensitive to two different wavelengths were stacked together and lenses were recorded in each layer. Initially, a theoretical design was developed in order to determine the recording conditions needed to achieve an efficient element. An acriflavine-sensitized photopolymer material was characterized so as to find the recording conditions for the expected range of spatial frequencies. Holographic lenses were then recorded in individual layers at blue and green wavelengths (in the appropriately sensitized photopolymer), which demonstrated uniform and high efficiency at the optimized exposure energies. Dual-wavelength sensitive stacks were then assembled and the sequential recording of stacked lenses was carried out. A set of four stacked lenses were recorded and tested by probing by a He-Ne laser at a wide range of angles for three positions across the element. The angular separation of +1 and -1 order maximum diffraction positions were analyzed. It was found that experimental angular separation matched well with theoretical values for the lenses. It was not possible to resolve the separate diffraction peaks from the blue and green lenses, as they were designed to coincide for blue and green wavelengths; even at $633 \mathrm{~nm}$, the angular separation is expected to be $<0.5^{\circ}$. Initial tests performed with cylindrically diverging beams showed strong diffraction into collimated off-axis beams for blue, green and white light. With some optimization, this approach could be used to develop stacked DOEs that can re-direct and collimate light with multiple wavelength ranges for potential applications in illumination, solar collection and displays.

Future work will involve investigating the dual-wavelength layer stacking approach for lenses with different off-axis angles and numerical apertures as well as a more detailed study of the independent photonic structures recorded by this method by deliberately separating the positions of the diffraction peaks.

Author Contributions: S.K. carried out most of the experimental work and developed aspects of the methodology as well as preparing the original draft. He acquired the funding for the work as part of a PhD scholarship. B.R. contributed to the experimental work in the final section. K.M. helped develop the methodology and contributed to draft preparation and data analysis. He is a co-supervisor of this work. K.R. assisted with the modeling and data analysis. I.N. helped develop the concept, methodology and materials. She is a co-supervisor of this work. S.M. helped develop the concept methodology and materials. She is the lead-supervisor of this work. All supervising authors contributed to reviewing and editing. All authors have read and agreed to the published version of the manuscript.

Funding: This research was funded by TU Dublin Fiosraigh PhD Scholarship fund. This research received no funding external to TU Dublin.

Acknowledgments: The authors would like to acknowledge Vincent Toal for his advice and the FOCAS research Institute for facilities and support.

Conflicts of Interest: The authors declare no conflict of interest. 


\section{References}

1. Kogelnik, H. Coupled Wave Theory for Thick Hologram Gratings. Bell Syst. Tech. J. 1969, 48, 2909-2947. [CrossRef]

2. Close, D.H. Holographic Optical Elements. Opt. Eng. 1975, 14, 408-419. [CrossRef]

3. Motogaito, A.; Machida, N.; Morikawa, T.; Manabe, K.; Miyake, H.; Hiramatsu, K. Fabrication of a binary diffractive lens for controlling the luminous intensity distribution of LED light. Opt. Rev. 2009, 16, 455-457. [CrossRef]

4. Mohammad, N.; Meem, M.; Wan, X.; Menon, R. Full-color, large area, transmissive holograms enabled by multi-level diffractive optics. Sci. Rep. 2017, 7, 1-6. [CrossRef] [PubMed]

5. Baldry, I.; Bland-Hawthorn, J.; Robertson, J.G. Volume Phase Holographic Gratings: Polarization Properties and Diffraction Efficiency. Publ. Astron. Soc. Pac. 2004, 116, 403-414. [CrossRef]

6. Bruder, F.K.; Fäcke, T.; Rölle, T. The Chemistry and Physics of Bayfol ${ }^{\circledR}$ HX Film Holographic Photopolymer. Polymers $2017,9,472$. [CrossRef]

7. Martin, S.; Akbari, H.; Keshri, S.; Bade, D.; Naydenova, I.; Murphy, K.; Toal, V. Holographically Recorded Low Spatial Frequency Volume Bragg Gratings and Holographic Optical Elements. Hologr. Mater. Opt. Syst. 2017. [CrossRef]

8. Wang, H.; Wang, J.; Liu, H.; Yu, D.; Sun, X.; Zhang, J. Study of effective optical thickness in photopolymer for application. Opt. Lett. 2012, 37, 2241-2243. [CrossRef]

9. Bianco, G.; Ferrara, M.A.; Borbone, F.; Roviello, A.; Striano, V.; Coppola, G. Photopolymer-based volume holographic optical elements: Design and possible applications. J. Eur. Opt. Soc. Rapid Publ. 2015, 10, 15057. [CrossRef]

10. Marín-Sáez, J.; Atencia, J.; Chemisana, D.; Collados, M.-V. Characterization of volume holographic optical elements recorded in Bayfol HX photopolymer for solar photovoltaic applications. Opt. Express 2016, 24, A720-A730. [CrossRef]

11. Orselli, E.; Zanutta, A.; Bianco, A.; Karafolas, N.; Cugny, B.; Sodnik, Z.; Fäcke, T. Photopolymer materials for volume phase holographic optical elements. In Proceedings of the International Conference on Space Optics Vol. 10562-ICSO 2016, Biarritz, France, 18-21 October 2016.

12. Han, J.; Liu, J.; Yao, X.; Wang, Y. Portable waveguide display system with a large field of view by integrating freeform elements and volume holograms. Opt. Express 2015, 23, 3534-3549. [CrossRef]

13. Khan, M.S.; Rahlves, M.; Lachmayer, R.; Roth, B. Polymer-based diffractive optical elements for rear end automotive applications: Design and fabrication process. Appl. Opt. 2018, 57, 9106-9113. [CrossRef]

14. Marín-Sáez, J.; Keshri, S.; Naydenova, I.; Murphy, K.; Atencia, J.; Chemisana, D.; Garner, S.; Collados, M.V.; Martin, S. Stacked holographic optical elements for solar concentration. In Proceedings of the Photonics Ireland Conference, Cork, Ireland, 3-5 September 2018.

15. Piao, M.L.; Kwon, K.C.; Kang, H.J.; Lee, K.Y.; Kim, N. Full-color holographic diffuser using time-scheduledmiterative exposure. Appl. Opt. 2015, 54, 5252-5259. [CrossRef]

16. Shen, Z.; Lan, T.; Wang, L.; Ni, G. Color demultiplexer using angularly multiplexed volume holograms as a receiver optical end for VLC based on RGB white LED. Opt. Commun. 2014, 333, 139-145. [CrossRef]

17. Avayu, O.; Almeida, E.; Prior, Y.; Ellenbogen, T. Composite functional metasurfaces for multispectral achromatic optics. Nat. Commun. 2017, 8, 14992. [CrossRef] [PubMed]

18. Hong, K.; Yeom, J.; Jang, C.; Hong, J.; Lee, B. Full-color lens-array holographic optical element for three-dimensional optical see-through augmented reality. Opt. Lett. 2014, 39, 127-130. [CrossRef]

19. Malallah, R.; Li, H.; Qi, Y.; Cassidy, D.; Muniraj, I.; Al-Attar, N.; Sheridan, J.T. Improving the uniformity of holographic recording using multi-layer photopolymer: Part II Experimental results. J. Opt. Soc. Am. A 2019, 36, 334-344. [CrossRef] [PubMed]

20. Meka, C.; Jallapuram, R.; Naydenova, I.; Martin, S.; Toal, V. Development of a panchromatic acrylamide-based photopolymer for multicolor reflection holography. Appl. Opt. 2010, 49, 1400-1405. [CrossRef] [PubMed]

21. Mahamat, A.H.; Narducci, F.A.; Schwiegerling, J. Design and optimization of a volume-phase holographic grating for simultaneous use with red, green, and blue light using unpolarized light. Appl. Opt. 2016, 55, 1618-1624. [CrossRef]

22. Jauncey, G.E.M. The Scattering of X-Rays and Bragg's Law. Proc. Natl. Acad. Sci. USA 1924, 10, 57-60. [CrossRef]

23. Moharam, M.G.; Young, L. Criterion for Bragg and Raman-Nath diffraction regimes. Appl. Opt. 1978, 17, 1757-1759. [CrossRef] [PubMed]

24. Keshri, S.; Murphy, K.; Toal, V.; Naydenova, I.; Martin, S. Development of a photopolymer holographic lens for collimation of light from a green light-emitting diode. Appl. Opt. 2018, 57, E163-E172. [CrossRef] [PubMed]

25. Akbari, H.; Naydenova, I.; Martin, S. Using acrylamide-based photopolymers for fabrication of holographic optical elements in solar energy applications. Appl. Opt. 2014, 53, 1343-1353. [CrossRef] [PubMed]

26. Pramitha, V. A New Metal Ion Doped Panchromatic Photopolymer for Holographic Applications. Ph.D. Thesis, Cochin University of Science and Technology, Cochin, India, November 2011. Available online: dyuthi.cusat.ac.in/purl/2357 (accessed on 1 May 2021).

27. Meka, C. Development of Acrylamide Based Photopolymer for Full Colour Display Holography. Ph.D. Thesis, Technological University Dublin, Dublin, Ireland, 2010. [CrossRef] 